\title{
Analysis of Ultrasonic Pulse Generated by Piezoelectric Material (LiNbO3 Cut Y-X) Using SVM Classifier
}

\author{
Hafdaoui Hichem ${ }^{1}$; Benatia Djamel ${ }^{2}$ \\ ${ }_{1,2}$ Electronics Department, Faculty of Technology, University of Batna 2, (Mostefa Benboulaid),05000, Algeria \\ ${ }^{1}$ Renewable Energy Development Centre, Bouzareah 16340, Alger, Algeria \\ ${ }^{1}$ Corresponding author: h.hafdaoui@cder.dz
}

\begin{abstract}
In this paper, we propose a new numerical method for ultrasonic pulse detection of an acoustics microwaves signal during the propagation of acoustics microwaves generated by piezoelectric substrate LiNbO3 Cut Y-X in ultrasonic transducer. We have used the classifications by support vector machines (SVM), the originality of this method is it provides the accurate values and help us to identify undetectable waves that we can not identify with the classical methods; in which we classify all the values of the real part and the imaginary part of the coefficient attenuation with the acoustic velocity in order to build a model from which we note the Ultrasonic Pulse or microwaves acoustics (bulk waves ). By which we obtain accurate values for each of the coefficient attenuation and acoustic velocity. This study will be very interesting in modeling and realization of acoustics microwaves devices (ultrasound) based on the propagation of acoustics microwaves.
\end{abstract}

Keywords - Ultrasonic ; Piezoelectric material ; Support Vector Machines (SVM); Classification

\section{INTRODUCTION}

Medical diagnostic ultrasound is an imaging modality that makes images showing a slice of the body, so-called tomographic images (tomo $=$ Gr. tome, to cut and graphic $=$ Gr. graphein, to write). It is a diagnostic modality, meaning that it gathers information about the biological medium without modification of any kind (Figure 1) the ultrasonic transducer is the one responsible for generating ultrasound and recording the echoes generated by the medium. Since the transducer should make mechanical vibrations in the megahertz range, a material that can vibrate that fast is needed. Piezoelectric materials are ideal for this. (Figure 2)

The interest in the use of piezoelectric materials as the wave propagation medium, lies in the propagation of appearance. These waves, in this case, spread in a resilient part (or Ultrasonic Pulse) and power; hence the name electro elastic or electroacoustic [1]. This coupling allows the piezoelectric waves that propagate acoustic velocities to be very stable with frequencies. The resulting material will be considered a delay structure and allows an efficient signal processing [2].

So the processed signal is applied to the electrodes of the transducer which in turn leads to deformation (compression and expansion) and a piezoelectric wave arises is propagating in the X direction (Figure 2) [2][3][4].

In our case, we propose another approach for the modelling of the acoustic microwaves with a complementary vision to the literature mentioned above. In this approach we interested especially in the detection of ultrasonics by the use of a (SVM) as detection tool in order to mark the mode of a ultrasonics.

In this work, we searched for all Acoustic velocity and Attenuation coefficients relying on fundamental relations and we classified all the values so that we can detect Ultrasonic Pulse easily.

\section{PhenOMENOLOGICAL TENSORIAL PIEZOELECTRIC EQUATIONS}

The signal to be treated will be applied to the electrodes of the transducer that generates the distortions (compression and dilatation), so a piezoelectric wave is generated and propagated in the $X$ direction. We consider the space coordinates as follows:

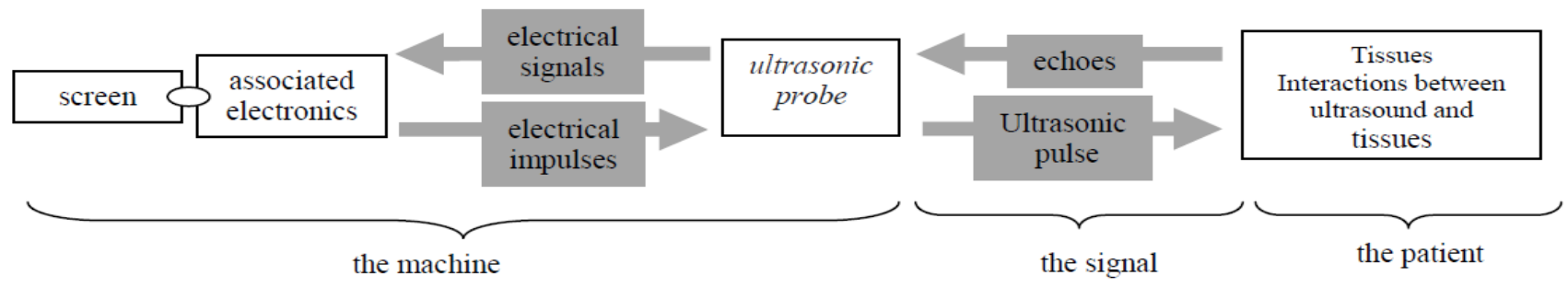

Figure 1. principle of ultrasound diagnosis 


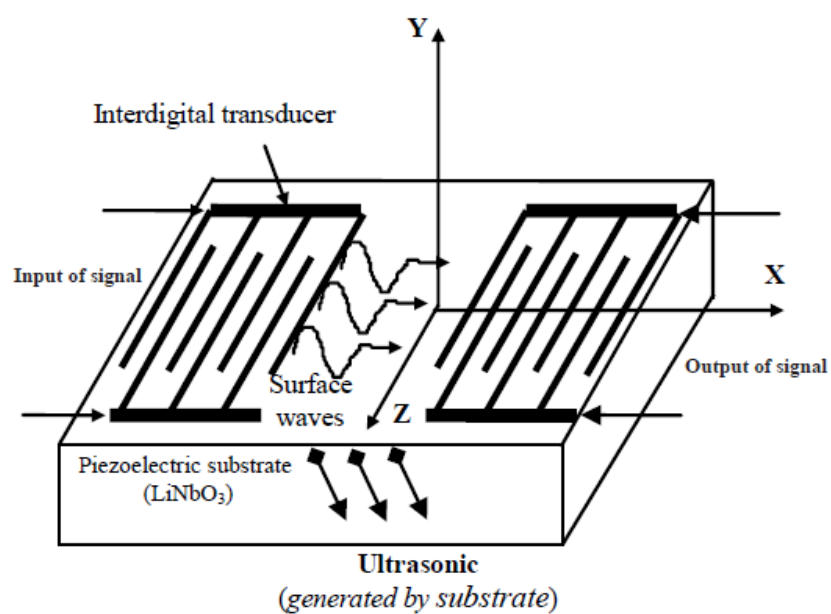

Figure 2. Ultrasonic Pulse generated by LiNbO3 substrate

$X=X_{1}, Y=X_{2}, Z=X_{3}$

The mechanical state of the medium is defined by two magnitudes of tensorial type, the stress Tij and the mechanical deformation (Strain) $\operatorname{Sij}(i, j=1,2,3)$.

The electric state of the medium is defined by two vectors, the electric field $E_{k}$ and the electric induction $D_{i}$. The stress tensor and the electric induction are defined like follows [5]:

$$
\begin{gathered}
T_{i j}=C_{i j k l} S_{k l}-e_{k i j} E_{k} \\
D_{i}=e_{j k l} S_{k l}+\varepsilon_{i k} E_{k}
\end{gathered}
$$

with $i, j, k, l=1,2,3$, where

$\varepsilon_{i k}$ : permittivity tensor $(\mathrm{F} / \mathrm{m})$

$e_{j k l}$ : piezoelectric tensor $(\mathrm{c} / \mathrm{m})$

$C_{i j k l}$ : elastic tensor $\left(\mathrm{N} / \mathrm{m}^{2}\right)$

The strain is bound to the relative displacements of the particles of the material environment is defined by :

$$
S_{i j}=\frac{1}{2}\left(\frac{\partial U_{i}}{\partial X_{j}}+\frac{\partial U_{j}}{\partial X_{i}}\right)
$$

where $U_{i}$ represents the elastic displacement of the particle $(i=1,2,3)$.

Note that, in the quasi-static approximation, an electric field of component can be defined by [6,7]:

$$
\mathrm{E}_{\mathrm{i}}=-\frac{\partial \mathrm{U}_{4}}{\partial \mathrm{X}_{\mathrm{i}}}
$$

where $U_{4}$ is the electric potential (with $i=1,2,3$ )

On the other hand, in the quasi-static approximation, the Maxwell's equation amounts to the Poisson's equation [5-7].

$$
\operatorname{div} \vec{D}=\frac{\partial D_{i}}{\partial X_{i}}=0
$$

The movement of the particles under the action of stress (constraints), is described by the following differential equation (Newton's 2nd law) [8-9]

$$
\nabla \mathrm{T}=\rho \frac{\partial^{2} U j}{\partial \mathrm{t}^{2}}
$$

where $\rho$ is the mass density of medium.

Replacing (3) and (4) in (1) and (2), we obtain

$$
\begin{aligned}
& \mathrm{T}_{\mathrm{ij}}=\mathrm{C}_{\mathrm{ijkl}} \cdot \frac{1}{2}\left(\frac{\partial \mathrm{U}_{\mathrm{j}}}{\partial \mathrm{X}_{\mathrm{k}}}+\frac{\partial \mathrm{U}_{\mathrm{k}}}{\partial \mathrm{X}_{\mathrm{j}}}\right)+\mathrm{e}_{\mathrm{kij}} \cdot \frac{\partial \mathrm{U}_{4}}{\partial \mathrm{X}_{\mathrm{k}}} \\
& \mathrm{D}_{\mathrm{ij}}=\mathrm{e}_{\mathrm{ikl}} \cdot \frac{1}{2}\left(\frac{\partial \mathrm{U}_{\mathrm{j}}}{\partial \mathrm{X}_{\mathrm{k}}}+\frac{\partial \mathrm{U}_{\mathrm{k}}}{\partial \mathrm{X}_{\mathrm{j}}}\right)-\varepsilon_{\mathrm{ik}} \cdot \frac{\partial \mathrm{U}_{4}}{\partial \mathrm{X}_{\mathrm{k}}}
\end{aligned}
$$

Replacing (7) and (8) in (5) and (6), we obtain

$$
\begin{gathered}
C_{i j k l} \frac{\partial^{2} u_{k}}{\partial X_{i} \partial X_{l}}+e_{l i j} \frac{\partial^{2}}{\partial X_{k} \partial X_{i}}=\rho \frac{\partial^{2} U_{j}}{\partial t^{2}} \\
e_{i k l} \frac{\partial^{2} U_{k}}{\partial X_{i} \partial X_{l}}-\varepsilon_{i k} \frac{\partial^{2} U 4}{\partial X_{k} \partial X_{i}}=0
\end{gathered}
$$

Equations (9) and (10) are the piezoelectric tensorial equations, they characterize electroelastic coupling between the elastic displacement vector of components $U_{1}, U_{2}, U_{3}$ and the electric potential $U_{4}$.

\section{THE Form OF SOLUTION}

The piezoelectric wave is the solution of the elastic and electric equations that satisfy elastic and electric boundary conditions. When these waves attenuate exponentially inside the piezoelectric material, they are known as surface waves and if this is not the case they are known as bulk waves.

Consider the following form of the surface wave (partial wave)

$$
\mathrm{U}_{\mathrm{i}}=\mathrm{u}_{\mathrm{i}} \exp \left(\mathrm{j} \beta \alpha_{\mathrm{i}} \mathrm{Y}\right) \exp -\mathrm{j}(\omega \mathrm{t}-\beta(1+\mathrm{j} \gamma) \mathrm{X})
$$

where $u i(i=1,2,3)$ are the displacement amplitudes, $u_{i}(i=4)$ is the amplitude of the electric potential, $k$ is the constant of propagation, the $\alpha_{i}$ are the penetration coefficients of the wave inside the piezoelectric substrate, $\gamma$ is the coefficient of longitudinal attenuation and $\omega$ is the angular pulsation.

We will be interested in the coefficient $\alpha_{\mathrm{i}}$. With a chosen YXcut LiNbO3 (Lithium Niobate).

Equations (9) and (10) can be written in a matrix form as

$$
[A][U]=[0]
$$

where $[\mathrm{A}]$ is a matrix $(4 \times 4)$, the elements of this matrix depend on the features of the piezoelectric material $\left(C_{i j k l}, \varepsilon_{i k}\right.$ and $\left.e_{l i j}\right)$ as well as of penetration coefficient $\alpha$ and of the acoustic velocity $V_{S}$.

The determinant of the matrix $[\mathrm{A}]$ must be zero to ensure a non-trivial solution, it can be written as

$$
\sum_{i=0}^{8} \beta_{i} \alpha^{i}=0
$$


where $B_{i}$ depends on the features of the piezoelectric material $\left(C_{i j k l}, \varepsilon_{i k}\right.$ and $\left.e_{l i j}\right)$ and of the acoustic velocity $V_{S}$.

The determinant of the matrix $[\mathrm{A}]$ must be zero, we have eight complex roots $(i=1, \ldots, 8)$ :

$$
\alpha_{i}=a_{i}+j b_{i}
$$

where $a_{i}$ is the real part and $b_{i}$ is the imaginary part, with $a_{m}=a_{m+1}$ and $b_{m}=b_{m+1}$

where $m=1,3,5,7$.

In the surface mode (or Rayleigh wave) the $\alpha_{\mathrm{i}}(i=1, \ldots, 4)$ are conjugated by pairs and only the complex roots with negative imaginary part are taken into consideration (for convergence reasons).

Let us first neglect the longitudinal attenuation $(\gamma=0)$ and insert (14) in (11) to obtain

$$
\mathrm{U}_{\mathrm{i}}=\mathrm{u}_{\mathrm{i}} \exp -\left(b_{\mathrm{i}} \mathrm{k} . \mathrm{Y}\right) \exp -\mathrm{j}\left[\omega \mathrm{t}-k\left(\mathrm{X}+a_{i} Y\right)\right.
$$

If we go inside the crystal ( $\mathrm{Y}$ tends to $-\infty$ ), the wave $U_{i}$ tends to zero. This corresponds to surface acoustic waves (SAW) .In the opposite case ( $\mathrm{Y}$ tends to $+\infty$ ), $U_{i}$ tends to infinity $(+\infty)$ (without physical signification).

\section{Remarks}

$$
\checkmark \text { If } \alpha_{r e}^{(i)}=0 \text { and } \alpha_{i m}^{(i)}>0 \text {, ultrasonic pulse will be }
$$
obtained and for a good detection of ultrasonic pulse we should have (Real part equals zero and the maximum positive value of Imaginary part (table 1)

\section{SuPPort Vector MACHINE (SVM)}

Support Vector Machine (SVM) is one the relatively modern methods that has showed high efficiently in recent years compared to older methods for classification including perceptron nervous systems. Work basis of SVM classification is data linearly classification and in linear division, we try to select a line that has more reliable edge (Figure 3).

To solve this equation, we should find optimum line for data by quadratic programming QP methods that are determined methods to solve limited problems. Before line division, we take data to higher dimensions via phi function to classify data with higher complexity in machine. We apply Lagrange Duality Theorem to transform minimization problem to duality form where we use simple function called core function that is vector coefficient of Phi function to solve a problem with very high dimensions. We can use different

core functions including exponential cores, vector and Sigmund [10-11] .

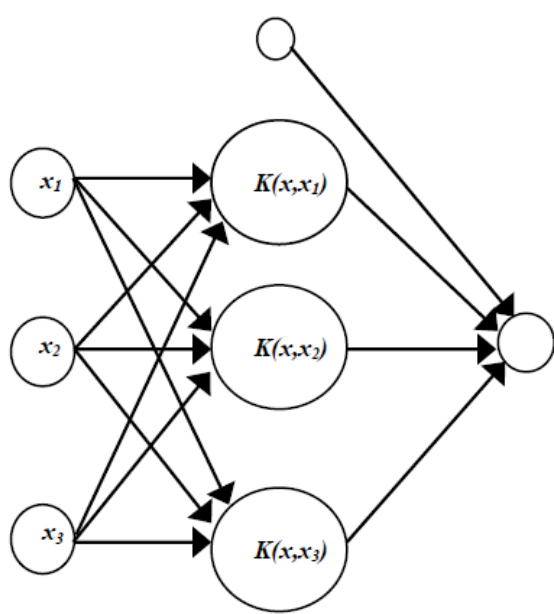

Figure 3.Architecture of SVM.

\section{RESUlts AND DisCUSSION}

Figure 4 shows the classification using SVM ; for each value of coefficient attenuation $\alpha 1$, we observe that Ultrasonic Pulse has 5 values, Figure 5 shows the classification using SVM but in this case the the classification factor is acoustic velocity Vs and this stag helps to know acoustic velocity Vs which corresponds to each value of coefficient attenuation $\alpha 1$, But in the attenuation $\alpha 2$ we note that ; Ultrasonic Pulse is not detected, (Figure 6), in this case we are not requiered to use the classification of acoustic velocity Vs, In the attenuation $\alpha 3$ we note that ; Ultrasonic Pulse is not detected (Figure 7) (Table 1), in this case we are not requiered to use the classification of acoustic velocity Vs. Figure 8 shows the classification using SVM ; for each value of coefficient attenuation $\alpha 4$, we observe that Ultrasonic Pulse has 4 values , Figure 9 shows classification for acoustic velocity corresponds to each value of coefficient attenuation $\alpha 4$.(Table 1)

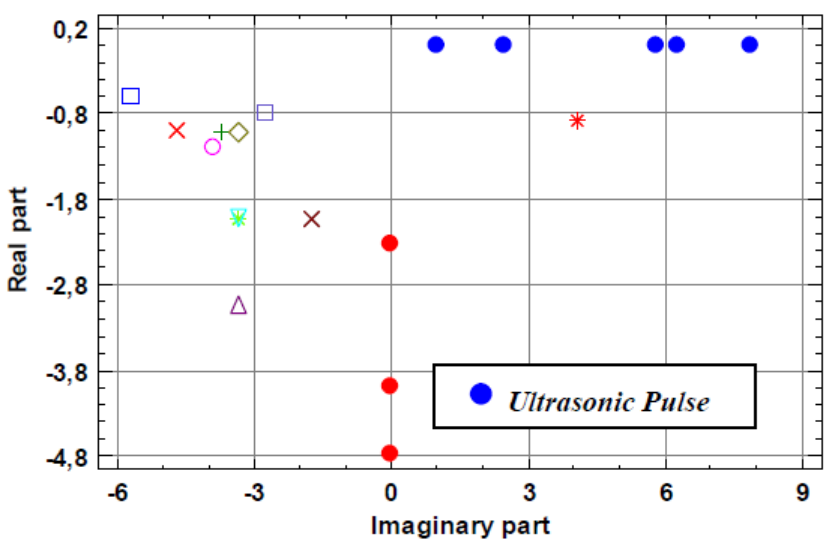

Figure 4: SVM classifier for $\alpha 1$. 


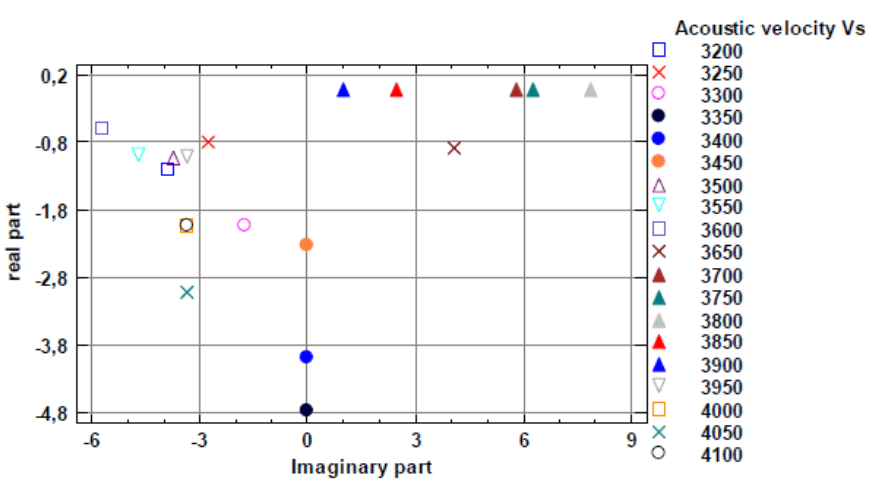

Figure 5. SVM classifier for $\alpha 1$ - Classification factor Vs

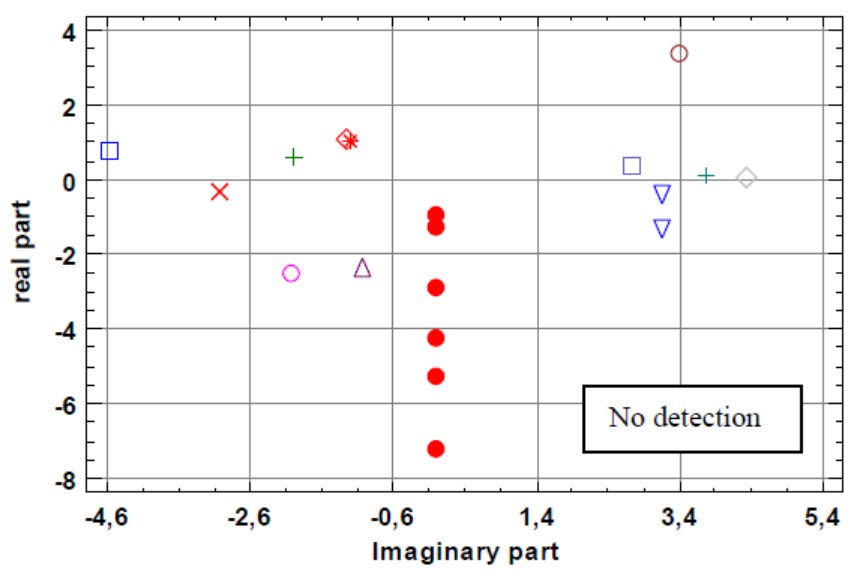

Figure 6. SVM classifier for $\alpha 2$.

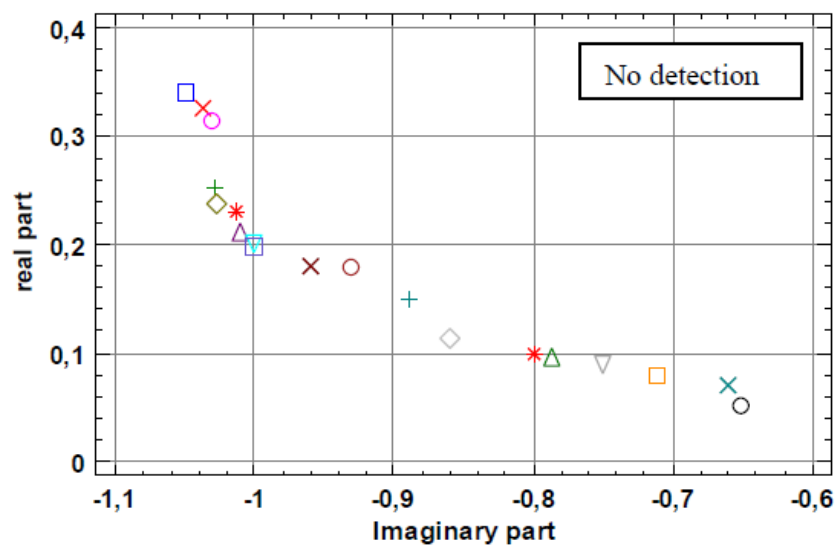

Figure 7: SVM classifier for $\alpha 3$.

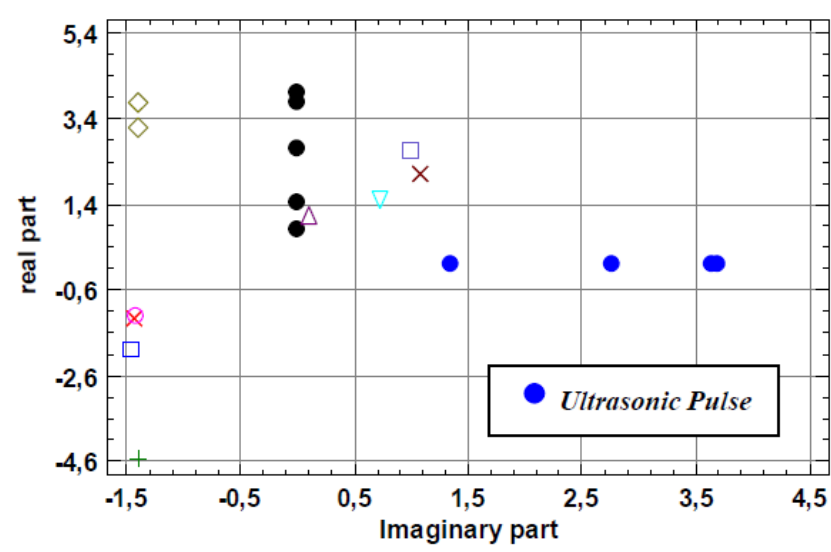

Figure 8: SVM classifier for $\alpha 4$.

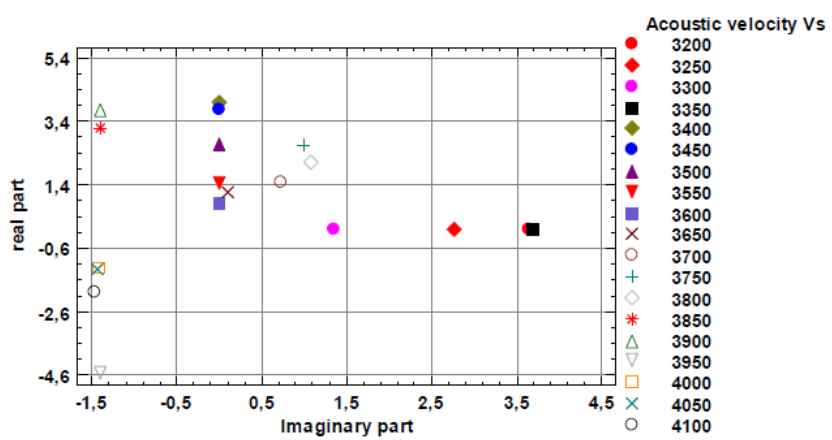

Figure 9: SVM classifier for a4- Classification factor Vs

TABLE I. SUMMARIZES THE FULL WORK

\begin{tabular}{|c|c|c|c|c|}
\hline $\begin{array}{l}\text { Coefficient } \\
\text { attenuation }\end{array}$ & Real part & $\begin{array}{c}\text { Imaginary } \\
\text { part }\end{array}$ & $\begin{array}{c}\text { Acoustic } \\
\text { velocity Vs } \\
(\mathrm{m} / \mathrm{s})\end{array}$ & $\begin{array}{c}\text { Ultrasonic } \\
\text { Pulse }\end{array}$ \\
\hline \multirow{5}{*}{$\alpha 1$} & 0 & 5,78765 & 3700 & Detection \\
\hline & 0 & 6,25443 & 3750 & Detection \\
\hline & 0 & 7,8765 & 3800 & $\begin{array}{c}\text { Good } \\
\text { Detection }\end{array}$ \\
\hline & 0 & 2,4543 & 3850 & Detection \\
\hline & 0 & 1,00001 & 3900 & Detection \\
\hline$\alpha 2$ & No values & No values & No values & No detection \\
\hline$\alpha 3$ & No values & No values & No values & No detection \\
\hline \multirow{4}{*}{$\alpha 4$} & 0 & 3,65 & 3200 & Detection \\
\hline & 0 & 2,765 & 3250 & Detection \\
\hline & 0 & 1,34571 & 3300 & Detection \\
\hline & 0 & 3,7 & 3350 & $\begin{array}{c}\text { Good } \\
\text { Detection }\end{array}$ \\
\hline
\end{tabular}

\section{CONCLuSION}

In this article, we explained that the phenomenon of Ultrasonic Pulse or acoustics bulk micrwaves are relying on numerical results at the level of attenuation coefficients. Changes in real and imaginary parts of the coefficients based on the acoustic velocity to detect these waves. It is quite clear that these waves are propagent into the piezoelectric material considered. Our method is applicable for any piezoelectric material, you just have to change the characteristics of the material parameters. The positive feature of this work gives us accurate values.

\section{REFERENCES}

[1] Royer, D., \& Dieulesaint, E. (1999). Elastic waves in solids II: generation, acousto-optic interaction, applications. Springer Science \& Business Media.

[2] Suchea, M., Christoulakis, S., Moschovis, K., Katsarakis, N., \& Kiriakidis, G. (2006). $\mathrm{ZnO}$ transparent thin films for gas sensor applications. Thin solid films, 515(2), 551-554.

[3] Ming, X., Lu, F., Liu, H., Chen, M., \& Wang, L. (2009). Formation and characterization of $\mathrm{ZnO}$ : $\mathrm{Tm}+$ optical waveguides fabricated by $\mathrm{Tm}+$ and $\mathrm{O}+$ ion implantation. Journal of Physics D: Applied Physics, 42(16), 165303. 
[4] Deng, M. (2001). Simulation of generation of bulk acoustic waves by interdigital transducers. In Ultrasonics Symposium, 2001 IEEE (Vol. 1, pp. 855-858). IEEE.

[5] Hafdaoui, H., Mehadjebia, C., \& Benatia, D. (2017, October). Using Probabilistic Neural Network (PNN) for Extracting Acoustic Microwaves (BAW) in Piezoelectric Material. In International Conference in Artificial Intelligence in Renewable Energetic Systems (pp. 308-315). Springer, Cham.

[6] Hichem, H., \& Djamel, B. (2018). A comparative study for two LiNbO3 cuts (YZ and YX) in detecting bulk acoustic microwaves using Probabilistic Neural Network. Engineering Science and Technology, an International Journal, 21(3), 527-531.

[7] Kurjak, A., \& Chervenak, F. A. (2017). Donald School Textbook of Ultrasound in Obstetrics \& Gynaecology. JP Medical Ltd.

[8] Lam, C. S., Gao, A., Lin, C. M., \& Zou, J. (2018, March). A review of Lamé and Lamb mode crystal resonators for timing applications and prospects of Lamé and Lamb mode PiezoMEMS resonators for filtering applications. In Proc. 7th Int. Symp. Acoustic Devices Future Mobile Commun. Syst (pp. 1-12).

[9] Rughoobur, G., Sugime, H., DeMiguel-Ramos, M., Mirea, T., Zheng, S., Robertson, J., ... \& Flewitt, A. J. (2018). Carbon nanotube isolation layer enhancing in-liquid quality-factors of thin film bulk acoustic wave resonators for gravimetric sensing. Sensors and Actuators B: Chemical, 261, 398-407.

[10] Yousofi, M. H., Yousofi, H., \& Razavi, S. A. M. (2014). Utilizing automatic recognition and classification of images for pattern recognition. International Journal of Intelligent Information Systems.

[11] Hafdaoui Hichem , Benatia Djamel .(2019) . Comparative between (LiNO3) and $(\mathrm{LiTaO} 3)$ in detecting acoustics microwaves using classification . IAES International Journal of Artificial Intelligence (IJAI), 8(1),33-43.

\section{Creative Commons Attribution License 4.0 (Attribution 4.0 International, CC BY 4.0)}

This article is published under the terms of the Creative Commons Attribution License 4.0

https://creativecommons.org/licenses/by/4.0/deed.en_US

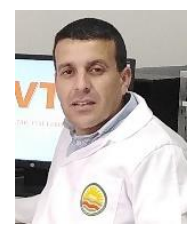

Hichem Hafdaoui he was born in Khenchela (Algeria) in 1984 . he got the licence diploma in electronic speciality communication that was in 2009 .by 2011 he got diploma master's degree in micro-waves and the devices of communication . 2016 he got diploma Doctorat field micro-waves and telecommunications in the University of Batna 2 . In 2020 he joined the Renewable Energy Development Centre as a permanent researcher. His research interests are: the propagation of acoustic microwaves in piezoelectric material.

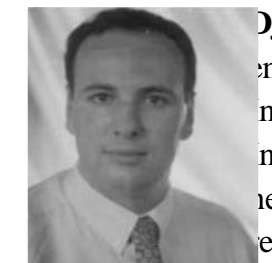

Jjamel Benatia, he was born in Constantine (Algeria) in 1967. He received his Engineering Diploma in electronic ngineering in 1990, a Master in communication in 1994 and the Ph.D in microwaves in 1999 from the Electronic nstitute, University of Constantine. In 1994 he joined the Electronic Department, University of Banta (Algeria). n 2000 he received the Associate Professor degree. In 2005 he received the Professor degree from 2003 to 2009 le has occupied the post of President of Scientist Comity of the Electronic Department of Batna University. His esearch interests are: the propagation of acoustic microwaves in piezoelectric material and the transmission by 